\title{
BMJ Open Biomarker discovery studies for patient stratification using machine learning analysis of omics data: a scoping review
}

\author{
Enrico Glaab (D) , ${ }^{1}$ Armin Rauschenberger (D) , ${ }^{1}$ Rita Banzi (D) , ${ }^{2}$ Chiara Gerardi (D) ," \\ Paula Garcia, ${ }^{3}$ Jacques Demotes (D) , ${ }^{3}$ the PERMIT Group
}

To cite: Glaab E,

Rauschenberger A, Banzi R, et al. Biomarker discovery studies for patient stratification using machine learning analysis of omics data: a scoping review. BMJ Open 2021;11:e053674. doi:10.1136/ bmjopen-2021-053674

- Prepublication history and additional supplemental materia for this paper are available online. To view these files, please visit the journal online (http://dx.doi.org/10.1136/ bmjopen-2021-053674).

Received 20 May 2021 Accepted 09 November 2021

A) Check for updates

C Author(s) (or their employer(s)) 2021. Re-use permitted under CC BY-NC. No commercial re-use. See rights and permissions. Published by BMJ.

${ }^{1}$ Luxembourg Centre for Systems Biomedicine, University of Luxembourg, Esch-sur-

Alzette, Luxembourg

${ }^{2}$ Center for Health Regulatory Policies, Istituto di Ricerche Farmacologiche Mario Negri IRCCS, Milano, Italy

${ }^{3}$ European Clinical Research Infrastructure Network, ECRIN, Paris, France

Correspondence to

Dr Enrico Glaab;

enrico.glaab@uni.lu

\section{ABSTRACT}

Objective To review biomarker discovery studies using omics data for patient stratification which led to clinically validated FDA-cleared tests or laboratory developed tests, in order to identify common characteristics and derive recommendations for future biomarker projects.

Design Scoping review.

Methods We searched PubMed, EMBASE and Web of Science to obtain a comprehensive list of articles from the biomedical literature published between January 2000 and July 2021, describing clinically validated biomarker signatures for patient stratification, derived using statistical learning approaches. All documents were screened to retain only peer-reviewed research articles, review articles or opinion articles, covering supervised and unsupervised machine learning applications for omics-based patient stratification. Two reviewers independently confirmed the eligibility. Disagreements were solved by consensus. We focused the final analysis on omics-based biomarkers which achieved the highest level of validation, that is, clinical approval of the developed molecular signature as a laboratory developed test or FDA approved tests.

Results Overall, 352 articles fulfilled the eligibility criteria. The analysis of validated biomarker signatures identified multiple common methodological and practical features that may explain the successful test development and guide future biomarker projects. These include study design choices to ensure sufficient statistical power for model building and external testing, suitable combinations of non-targeted and targeted measurement technologies, the integration of prior biological knowledge, strict filtering and inclusion/exclusion criteria, and the adequacy of statistical and machine learning methods for discovery and validation. Conclusions While most clinically validated biomarker models derived from omics data have been developed for personalised oncology, first applications for noncancer diseases show the potential of multivariate omics biomarker design for other complex disorders. Distinctive characteristics of prior success stories, such as early filtering and robust discovery approaches, continuous improvements in assay design and experimental measurement technology, and rigorous multicohort validation approaches, enable the derivation of specific recommendations for future studies.

\section{INTRODUCTION}

Personalised medicine is a rapidly developing area in healthcare research and practice,

\section{Strengths and limitations of this study}

- This scoping review provides an overview of biomarker discovery studies using machine learning analysis of omics data which have led to clinically validated diagnostic and prognostic tools.

- The review discusses shared characteristics of successful biomarker studies as a guidance for study design, discovery and validation method choices for future projects.

- Data extraction and analysis methods focus on deriving recommendations to optimise the design of prospective studies and improve analysis workflows for retrospective studies.

- The review applied minimum eligibility criteria for sample size and statistical validation, but did not assess the quality of the included studies.

which aims at providing more effective and safer therapies tailored to the individual patient, by exploiting subject-specific molecular, clinical and environmental data sources (box 1).

A central tool in personalised medicine and the focus of this study is the machine learning (ML) analysis of omics profiling data to derive molecular biomarker signatures for disease-based or drug-based patient stratification. ${ }^{1}$ The major goals for ML-based omics biomarker development are to develop more reliable and robust tests for drug response prediction, early diagnosis, differential diagnosis or prognosis of the future clinical disease course. ${ }^{2}$ Omics-derived biomarker signatures may help to guide treatment decisions, and to focus therapies on the right populations to prevent overtreatment, increase success rates and reduce costs. ${ }^{3}$ As a research and information tool, they may enable a better monitoring of disease progression and treatment success, and guide new drug development and discovery. ${ }^{4}$ In contrast to classical singlemolecule biomarker approaches, omics signatures have the potential to provide 


\section{Box 1 What is personalised medicine?}

According to the European Council Conclusion on personalised medicine for patients, personalised medicine is 'a medical model using characterisation of individuals' phenotypes and genotypes (eg, molecular profiling, medical imaging, lifestyle data) for tailoring the right therapeutic strategy for the right person at the right time, and/or to determine the predisposition to disease and/or to deliver timely and targeted prevention. ${ }^{116}$

In the context of the PERMIT project, we applied the following common operational definition of personalised medicine research: a set of comprehensive methods (methodology, statistics, validation, technology) to be applied in the different phases of the development of a personalised approach to treatment, diagnosis, prognosis or risk prediction. Ideally, robust and reproducible methods should cover all the steps between the generation of the hypothesis (eg, a given stratum of patients could better respond to a treatment), its validation and preclinical development, and up to the definition of its value in a clinical setting. ${ }^{19}$

more sensitive, specific and robust predictions of diseaseassociated outcomes. ${ }^{5}$

However, while biomarker discovery projects using omics data have already led to the successful development of clinically validated diagnostic and prognostic tests, ${ }^{6-15}$ many biomarker studies are discontinued after early development stages or fail in later clinical validation stages. Dedicated statistical and ML methodologies for omics biomarker discovery and validation have been published, as well as recommendations for study design, implementation and reporting. ${ }^{16} 17$ The distinctive features and approaches which characterise prior successes in translating omics research findings into clinically validated tests have, however, not yet been investigated in detail. In order to guide future projects on suitable method choices, there is a need for dedicated studies on the key determinants of previous translational successes in ML-based omics biomarker development.

As part of an EU project on 'Personalised Medicine Trials' $\left(\right.$ PERMIT $^{18}$ ), funded within the H2020 framework, we have therefore investigated the current methodological practices for personalised medicine, covering ML approaches for omics-based patient stratification as a major focus area. While a broader series of questions was established and examined for the overall scoping review, ${ }^{19}$ for this manuscript, we focused our analysis on biomarker discovery studies that have led to successful, clinically validated FDA-cleared tests or laboratory developed tests (LDTs), to determine their shared and distinctive characteristics compared with studies with no clinical translation. In particular, we aimed to address the following specific research questions:

- Which omics-derived biomarker discovery studies have led to clinically validated tests for patient stratification (LDTs or FDA-cleared tests)?

- What are the key characteristics shared by successful omics biomarker studies and distinguishing them from previously published biomarker studies which have not yet led to clinically validated tests?
- Which types of model building and validation methods have been used to develop clinically validated biomarker signatures, and what are the lessons learnt and recommended workflows?

- Which recommendations and guidelines have been proposed to address common challenges in biomarker development using omics data?

These questions lend themselves to a scoping review, because omics-derived biomarker development is still an evolving field, and a preliminary assessment of the potential scope and size of the available biomedical literature on these topics is required as a first step for further follow-up research. Therefore, the objective of this study was to address the above questions by retrieving and examining the current literature on biomarker discovery and validation studies using omics data and ML approaches. While the focus on articles describing discovery and validation approaches covers relevant aspects for clinical translation, we point out that other translational and regulatory aspects, such as the assessment of the clinical efficacy of biomarker-associated treatment decisions, the assessment of cost-effectiveness and research ethics, are not addressed in the present review, but have been discussed in previous dedicated articles. ${ }^{20-24}$ Our scoping review also does not aim at providing a quantitate benchmark evaluation of different ML approaches, but relevant studies have previously been presented for supervised $\mathrm{ML},{ }^{25}$ unsupervised clustering $^{26}$ and survival prediction ${ }^{27}$ on multiple omics data types.

\section{METHODS}

We conducted a scoping review following the methodological framework suggested by the Joanna Briggs Institute. ${ }^{28}$ This framework consists of six stages: (1) identifying the research questions, (2) identifying relevant studies, (3) study selection, (4) charting the data, (5) collating, summarising and reporting results and (6) consultation.

The scoping review approach was considered most suitable to respond to the broad scope and the evolving nature of the field. Compared with systematic reviews that aim to answer specific questions, scoping reviews present a general overview of the evidence pertaining to a topic and are useful to examine emerging trends, to clarify key concepts and identify gaps. ${ }^{29} 30$ Before conducting the review, a study protocol was published on the online platform Zenodo. ${ }^{19}$ Due to the iterative nature of scoping reviews, deviations from the protocol are expected and duly reported when occurred. We used the Preferred Reporting Items for Systematic reviews and Meta-Analyses extension for Scoping Reviews checklist to report our results $^{31}$ (online supplemental file 1).

\section{Study identification}

Relevant studies and documents were identified, balancing feasibility with breadth and comprehensiveness of searches. We searched PubMed, EMBASE and Web of Science (last search date: 27 July 2021) for articles 


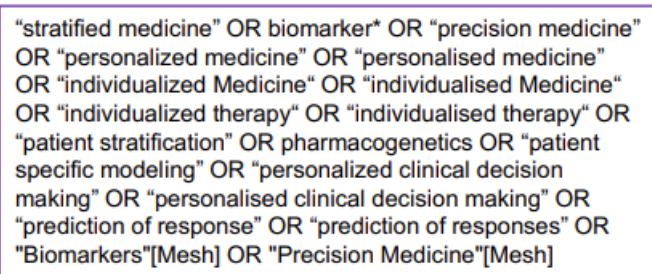

"Biomarkers"[Mesh] OR "Precision Medicine"[Mesh]

\section{Genomics"[Mesh]) OR "Metabolomics"[Mesh]) OR}

"Epigenomics"[Mesh]) OR "Microarray Analysis"[Mesh]) OR

"Mass Spectrometry"[Mesh] OR Omic* OR "omic based"

OR "multi omic" OR "multi omics" OR genomic* OR

transcriptomic* OR proteomic ${ }^{*}$ OR metabolomic* OR

lipidomic* OR epigenomic* OR microarray OR "RNA seq"

OR "mass spectrometry")

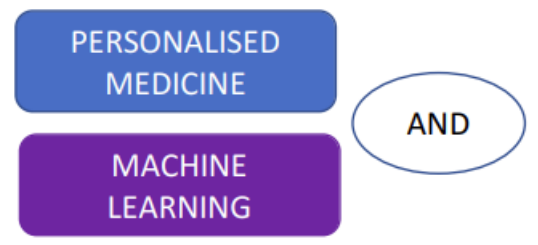

\section{OMICS}

\section{VALIDATION}

Validation Studies as Topic"[Mesh]) OR "Validation Study" [Publication Type] OR "Sensitivity and Specificity"[Mesh]) OR "Benchmarking"[Mesh]) OR validation OR validity OR validated OR "cross validation" "cross validated" OR "clinical utility*" OR accuracy OR robustness OR reliability* OR sensitivity OR specificity OR benchmark* OR bias OR "cross study" OR "cross studies")

Figure 1 Keyword based search strategy for the scoping review. Four categories of keywords were defined to retrieve relevant articles from the biomedical literature on machine learning analyses of omics data for personalised medicine, which include a validation study (highlighted by the coloured boxes in the centre). For each category relevant keywords were determined, including controlled vocabulary terms from the Medical subject Headings (MeSH) thesaurus by the US National Library of Medicine (upper and lower boxes with frames coloured according to the corresponding category). As indicated by the keyword 'and' in the centre, a conjunctive search was conducted, that is, every retrieved article had to contain at least one keyword from each category. This strategy was adapted for searching the other databases.

describing supervised or unsupervised ML analyses for biomarker discovery or personalised medicine, including both discovery and validation methods. The relevance of the search methodology was ensured by using a strict multistage filtering, considering only articles including at least one relevant search term per category from four categories of keywords ('Personalised medicine/ Biomarkers', 'Omics', 'Machine Learning' and 'Validation', covering both synonyms for these terms and closely related keywords, see figure 1, illustrating the keywordbased search strategy, and online supplemental file 2 for the detailed search queries), and subsequently postfiltering the retrieved articles manually to exclude studies not involving omics-based biomarker research or lacking a description of ML and validation analyses (see sections on Eligibility criteria and Study selection). To cover only relevant scientific content, the scope was limited to journal publications and meeting abstracts from international conferences and workshops, and no other grey literature was included. We restricted inclusion to reports published from January 2000 to July 2021 (covering also 'online first' articles with official publication date in the future) in English, French, Spanish, Italian and German language. Since to the best of our knowledge, the first clinically validated FDA-cleared omics-derived biomarker signature was published in 2002, ${ }^{32}$ only few preliminary discovery studies were expected to have taken place significantly earlier than 2002, and we, therefore, did not extent the search further backwards in time than January 2000.

\section{Eligibility criteria}

We included peer-reviewed methodology articles, review articles, opinion articles on supervised and unsupervised ML methods for omics disease prediction and stratification and associated statistical cross-validation (CV) and multicohort validation methods (addressing accuracy, robustness and clinical relevance). Only approaches tested on real-world biomedical omics data were reviewed, while studies relying purely on simulated data were excluded. We also excluded papers on biomarker methods without a demonstrated biomedical application, and those with insufficient sample size (ie, removing studies covering less than 50 samples per group for the main conditions studied, unless a dedicated power calculation was presented) or statistical validation (ie, lack of clear descriptions of CV or external testing methodology, performance metrics and test statistics). These exclusion criteria were not specified in the generic review protocol, but they were agreed among the authors prior to the screening process.

To cover both data from original research papers and prior systematic reviews, we extracted information from three main article types: (1) applied research papers, (2) methodology articles with demonstrated applications and (3) review articles on methods, applications and validation approaches.

Apart from these inclusion and exclusion criteria, for the final result presentation, the statistical investigations covered all selected articles, whereas the detailed discussion of study characteristics focused on the studies that 
led to clinically validated biomarker signatures tested on multiple cohorts with large sample sizes (ie, studies using a power calculation to demonstrate the adequacy of the chosen sample sizes, or covering hundreds or thousands samples per studied subject group).

\section{Study selection}

We exported the references retrieved from the searches into the online tool Rayyan. ${ }^{33}$ Duplicates were removed automatically using the reference manager Endnote V.X9 (Clarivate Analytics, Philadelphia, USA) and manually by the reviewers. One reviewer loaded the retrieved records into the online screening tool Rayyan, ${ }^{33}$ and two reviewers confirmed the eligibility independently by covering both the screening for all records and the full-text review for the articles preselected by the screening. Disagreements were solved by consensus.

\section{Charting the data and synthesis of results}

We designed a data extraction form using Excel (online supplemental file 3). General study characteristics extracted covered author names, title, citation, type of publication (eg, journal article, meeting abstract), study population and sample size (if applicable), methodology/ study design and outcome measures (if applicable). Specific items associated with the topic of the scoping review included the study type (eg, case-control study, differential diagnosis study, prognostic study, reviewmethods, review-applications, review-validation); the article type (journal or conference article), the generic ML domain (eg, supervised/unsupervised); and the name of specific approaches for outcome prediction and for validation. Moreover, to capture key findings related to the review questions, relevant sentences were extracted from each reviewed article, and if needed, complemented by a brief explanatory remark, and by writing out abbreviations used in the original text.

The reviewers piloted the data extraction form using five records from the retrieved article collection. Two reviewers (EG, AR) working independently extracted the data from the included articles. In the case of disagreements, consensus was obtained by discussion.

In the final full-text review stage, the preselected articles were grouped by topic, categorising articles into applied versus methodological studies, supervised versus unsupervised analyses and assigning algorithm type identifiers to each article (review articles and papers on validation methodologies were considered as separate categories without a specific algorithm type assignment). The fulltext review and categorisation of articles into different publication types was done through independent manual inspection by the two reviewers.

While the information on sample sizes and validation methods was documented as part of the data extraction (online supplemental file 4, a spreadsheet version has been made available on the online platform Zenodo ${ }^{34}$ ), it was not within the remit of this scoping review to assess

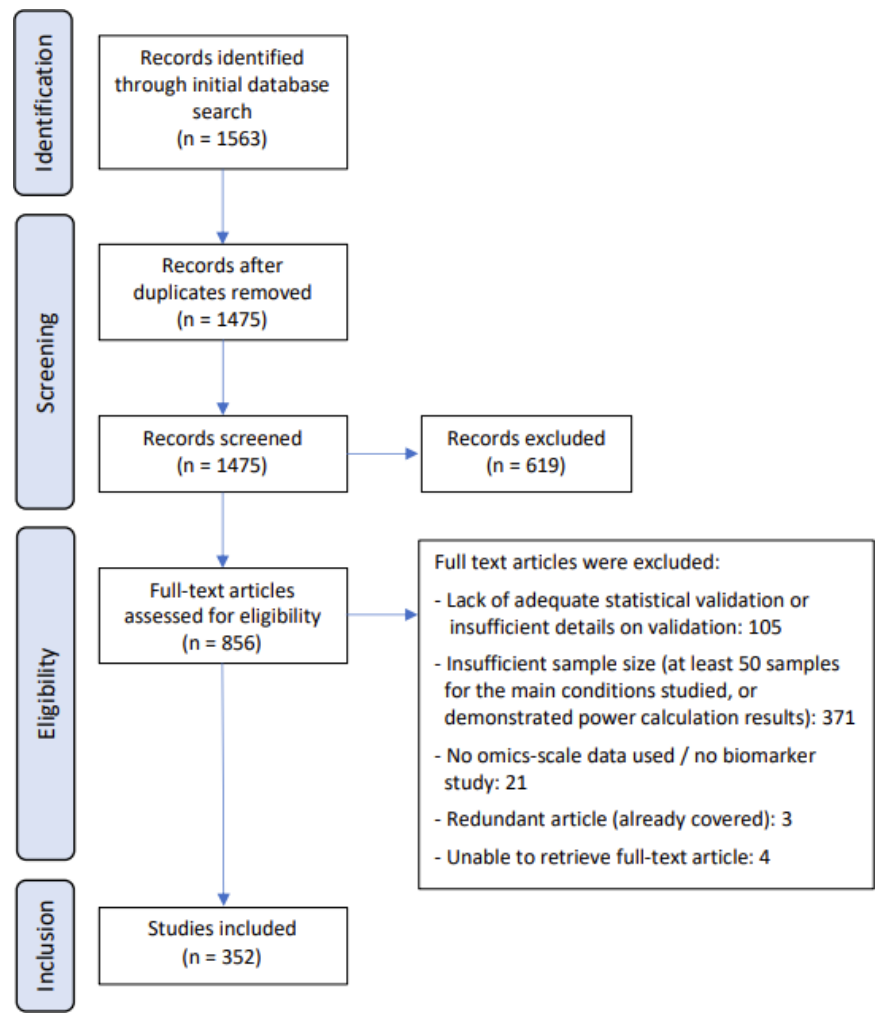

Figure 2 Study selection flow diagram. Flow diagram of the procedure for the scoping review article identification, screening, eligibility assessment and final inclusion, according to the PRISMA (Preferred Reporting Items for Systematic Reviews and Meta-Analyses) scheme. ${ }^{31}$ Reasons for excluding full-text articles were not mutually exclusive.

the methodological quality of individual studies included in the analysis.

\section{Consultation exercise}

The members of the PERMIT consortium, associated partners, and the PERMIT project Scientific Advisory Board discussed the preliminary findings of the scoping review in a 2-hour online workshop.

\section{Patient and public involvement}

The European Patients' Forum is a member of PERMIT project. Although not directly involved in the conduction of the scoping review, they received the draft review protocol for collecting comments and feedback.

\section{RESULTS}

\section{Study selection and general characteristics of reports}

We retrieved 1563 abstracts from the literature search. After the removal of duplicates, we screened the remaining 1475 abstracts for eligibility. A total of 619 records were excluded, while 856 abstracts were retained for the full-text assessment. Finally, we included 352 articles that passed all filtering criteria in the data extraction and analysis (see flow chart in figure 2 and online supplemental file 4, providing the reference for each selected article, as well as information on the study type and 


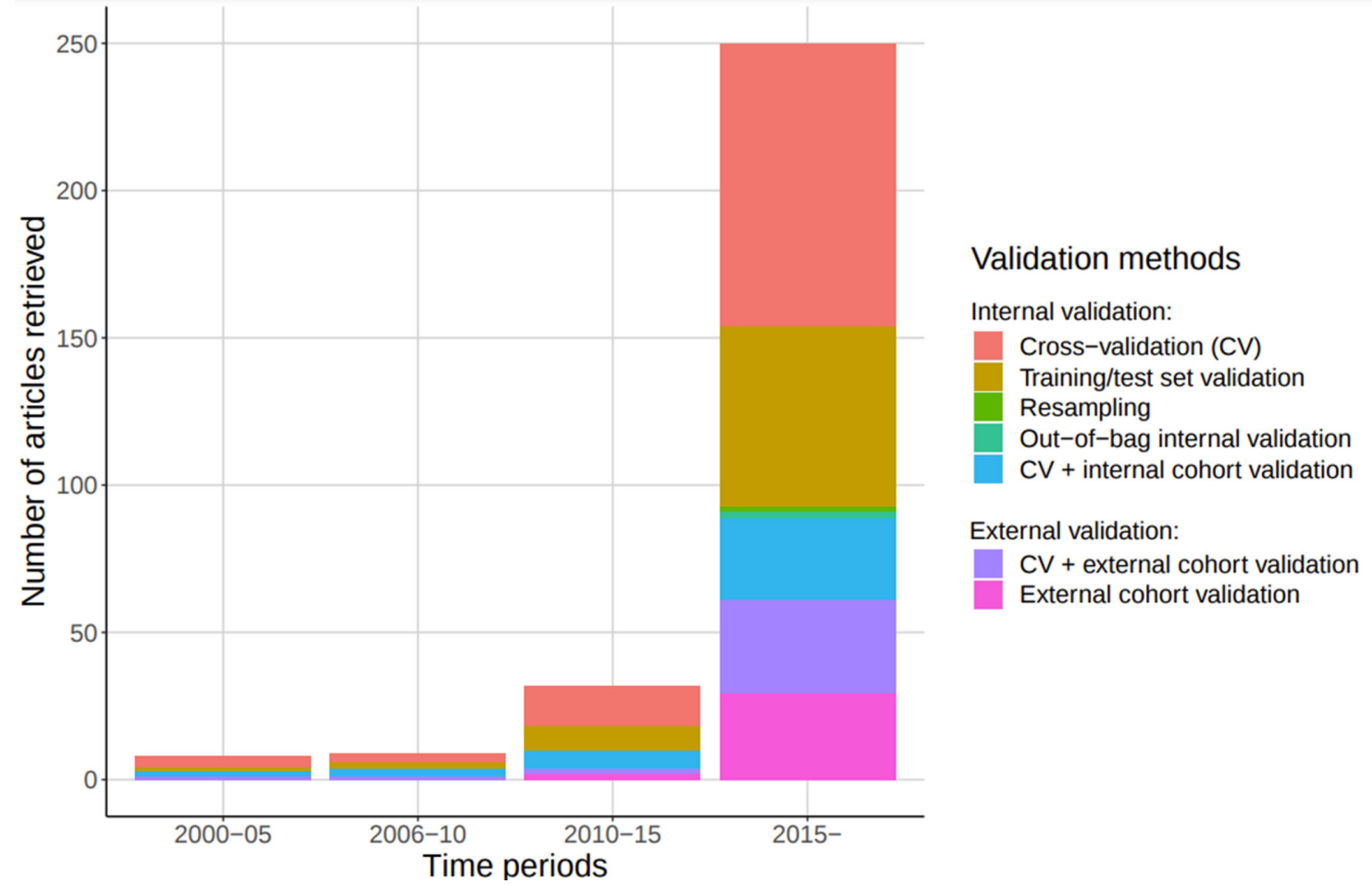

Figure 3 Validation methods used in omics biomarker studies. Stacked bar chart of the number of articles retrieved in the scoping review for different categories of validation methods used in the underlying biomarker studies (covering time periods from 2000 to 2021). The majority of studies use only internal cohort validation approaches, such as CV, training/test set split validation, resampling/bootstrapping-based validation, out-of-bag validation (for tree-based classifiers), and combinations of CV and test set validation within the same cohort. Studies with an external validation on an independent patient cohort (with or without an additional internal CV) are still underrepresented, even in more recent time periods. All filtered full-text articles derived from the scoping review except for review articles were included in the analysis.

methodology, the outcome measures, the validation type, and representative sentences from each article on the main study results and key findings; a spreadsheet version of this table has been made available on the online platform Zenodo ${ }^{34}$ ).

The full-text article review revealed that many studies did not meet the pre-defined inclusion criteria: 371 articles $(43 \%)$ were removed because of an insufficient sample size, and 105 further articles $(12 \%)$ were excluded because they provided insufficient details on the validation results or methodology (see figure 2). This shows that the challenges of recruiting an adequate number of participants per study group or conducting sufficient omics profiling experiments for robust model building and validation are not met in a large proportion of omics biomarker studies. Moreover, many studies lack adequate documentation for the study design and validation.

For the selected articles that cover primary research on omics biomarker studies, the majority $(78 \%)$ rely entirely on an internal validation involving data from only a single cohort, whereas studies that use an external validation on an independent cohort are still underrepresented (only 12\% of articles describe both an internal CV and an external cohort validation, and an additional $10 \%$ include an external validation, but do not report internal $\mathrm{CV}$ results). However, when comparing the numbers of published studies over different periods of time during the past 20 years, the relative proportion of studies including an external validation has increased in recent years (see figure 3), suggesting a growing recognition of the importance of independent, multicohort validation.

Next, we investigated the countries of origin for the selected articles, showing that the USA are contributing the largest proportion of validated biomarker studies (28\%), followed by China (18\%), Canada (5\%), Germany $(4 \%)$ and the UK and India (both 3\%; see also figure 4, providing a map visualisation of the country statistics). These country representations show limited correlation with population sizes and may largely reflect worldwide variation in relative biomedical research productivity reviewed in previous study. ${ }^{35}$ Since the most prolific countries in the development of molecular diagnostics have already set up policies and regulations for omics-based and ML-based in vitro diagnostics and medical devices (eg, see the life cycle regulation of artificial intelligence based and ML-based software devices in the USA ${ }^{36}$ ), they may also provide a role model for countries still in the process of establishing similar regulatory frameworks.

When inspecting the representation of study design types in the filtered article collection, the great majority of documents described diagnostic studies $(67 \%)$, prognostic and survival prediction studies were covered in $8 \%$ of articles, and studies examining therapy or drug response in $7 \%$ (see figure 5). Apart from this, 13\% of 

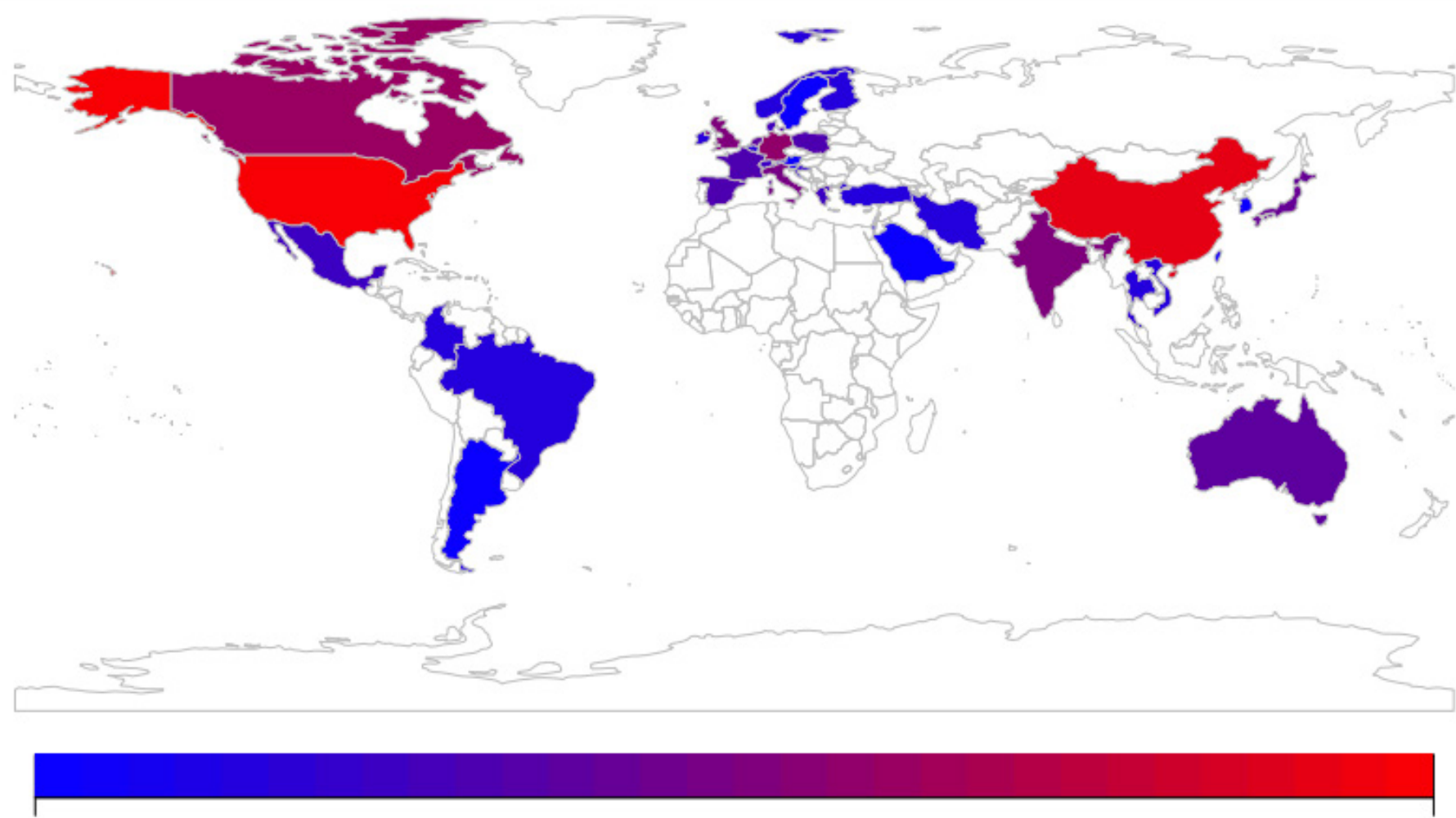

1

Figure 4 Map representation of country statistics for the selected articles. The number of articles originating from different countries among the studies selected in the full-text review are visualised on a world map representation using a colour gradient from blue ( 1 article) to red (98 articles=maximum contribution by a single country; using a logarithmic colour gradient scale to highlight differences over a broad value range).

articles were reviews on methodologies and applications in the field, and $5 \%$ of articles described other rare study types (eg, tissue-of-origin prediction studies or combinations of different study types).

Since a detailed discussion of all filtered articles is not within the scope of the present review, in the following,

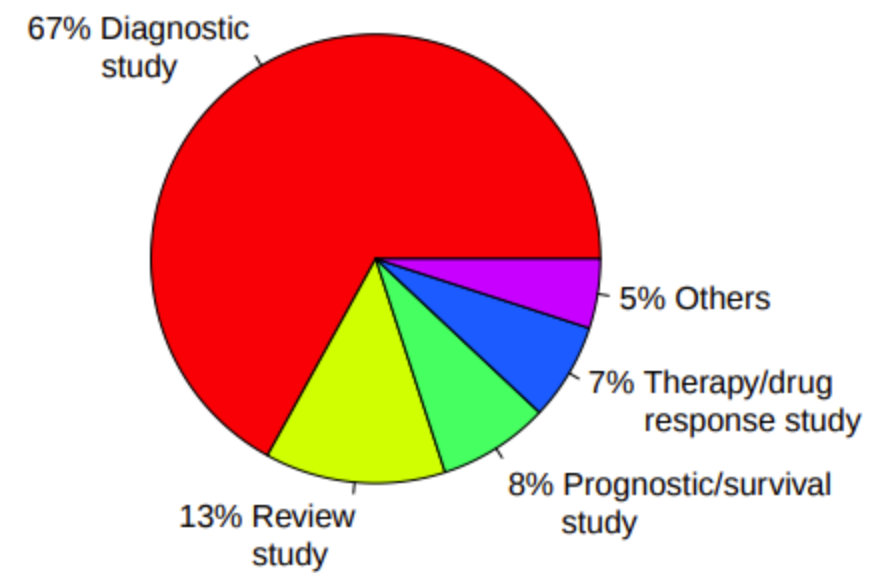

Figure 5 Representation of study types among the selected articles. The percentage of articles describing case-control studies, therapy/drug response studies, differential diagnosis studies, prognostic and survival prediction studies, as well as review studies and other study types is represented as a pie chart. we focus on reviewing representative omics biomarker studies which achieved the highest validation level, that is, clinical approval of the developed molecular signature as an LDT or FDA approved test (see the overview of studies in table 1 and the FDA web-site ${ }^{37}$ ). We investigate the shared features of these successful studies, examine how they address common shortcomings and missing features of other reviewed studies, and summarise the lessons learnt.

\section{Success stories in OMICs-based biomarker signature development}

Cancer approved omics-derived diagnostic tests (nine studies)

The first and most well-known omics-derived molecular test to receive FDA clearance was MammaPrint, a prognostic signature using the RNA expression activity of 70 genes to estimate the risk for distant tumours metastasis and recurrence in early-stage breast cancer patients. ${ }^{6}{ }^{32} 38-41$ This test was developed at the Netherlands Cancer Institute, using DNA microarray analysis to investigate primary breast tumours of 117 patients. Supervised ML was applied to the resulting data to identify a highly predictive gene signature for a short interval to distant metastases in lymph node negative patients. ${ }^{32}$

A distinctive feature of the development approach behind this signature in comparison to other reviewed studies was the multistage filtering and CV strategy used 
Table 1 Examples of clinically approved omics-derived diagnostic or prognostic tests designs applied to personalised medicine (synonyms for the same test are separated by the '/'-symbol)

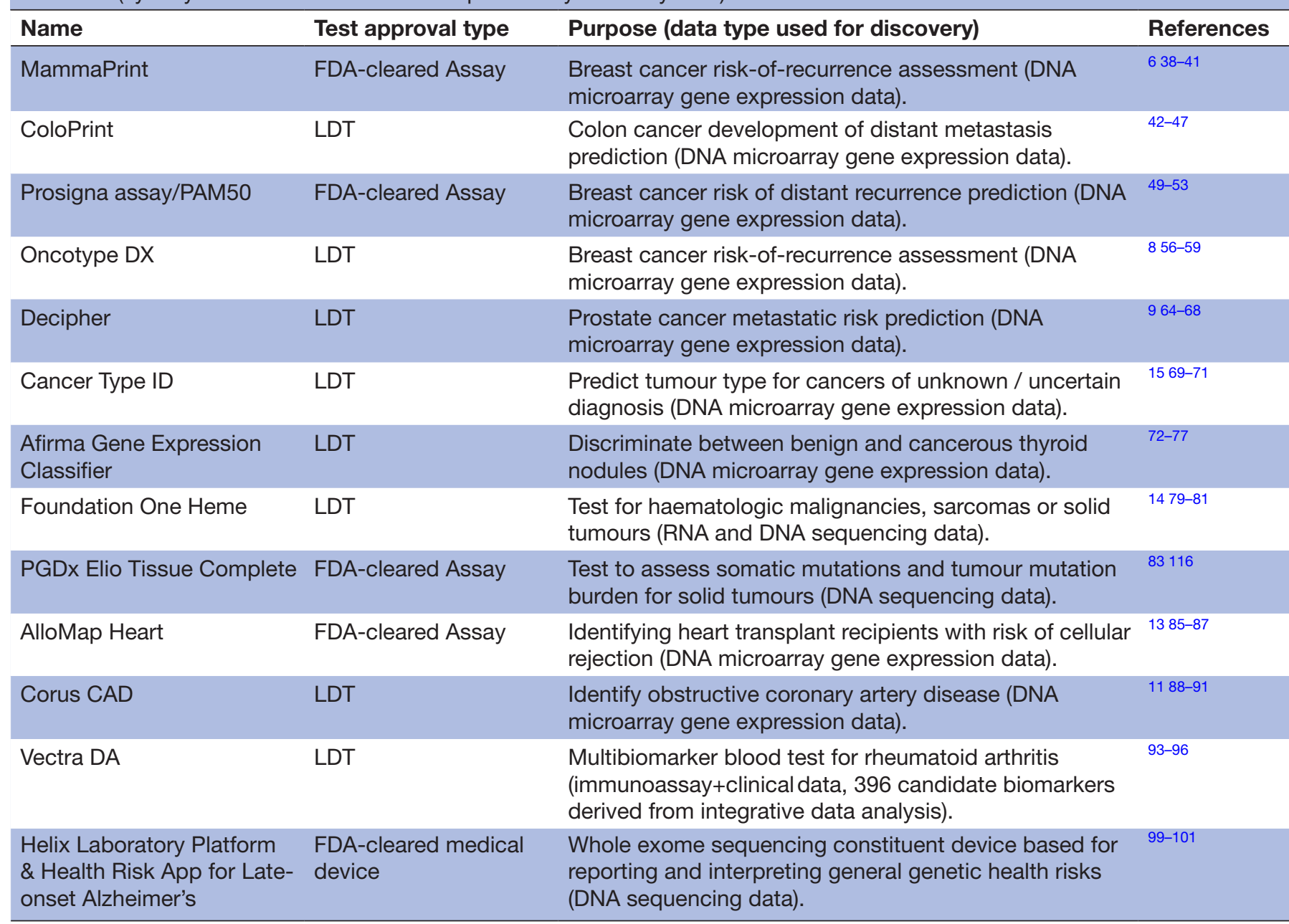

FDA approval status was checked on the web-site by the FDA (37) and reflects the status as of July 2021.

CAD, coronary artery disease; FDA, U.S. Food and Drug Administration; LDT, laboratory developed test.

in the initial discovery study, which may explain the repeated confirmation of the signature in later validation studies. ${ }^{68-41}$ From $25 \mathrm{k}$ genes represented on the DNA microarrays, only those significantly regulated in more than 3 tumours out of 78 sporadic lymph-node negative patients were preselected, and further filtered by retaining only the genes with a minimum absolute correlation with the disease outcome of 0.3 . The resulting list of 231 genes, rank-ordered by absolute correlation, was investigated by sequentially adding the next top five genes from the list to a candidate ML classifier and evaluating its performance by leave-one-out CV. This procedure was repeated as long as the estimated accuracy of the classifier improved, providing a final candidate signature of 70 genes. The final signature was validated on multiple independent test sets, including a set of 19 external samples in the original study and several additional validations on independent cohorts in follow-up studies. ${ }^{63-41}$

The MammaPrint signature provided the role model for the subsequent development of a similar prognostic test for colon cancer, ColoPrint. ${ }^{42-47}$ This test aims at detecting the approx. $20 \%$ of patients with stage II colon cancer expected to experience a relapse and develop distant metastases. It uses an 18-gene expression signature, developed by analysing DNA microarray data in a similar manner to the MammaPrint approach. The diagnostic approach has been commercialised as an LDT to assist physicians in selecting treatment options for colon cancer patients. Similar to MammaPrint, the signature development was characterised by extensive discovery and validation studies, which involved multiple statistical reproducibility, stability and precision analyses for independent, large-scale patient cohorts. ${ }^{48}$

Another widely used cancer-related LDT, which received clearance by the U.S. Food and Drug Administration (FDA) in 2013, is the Prosigna Breast Cancer Prognostic Gene Signature Assay, previously called PAM50 test. ${ }^{49-53}$ This assay assesses mRNA expression for a signature of 58 genes ( 50 target genes +8 endogenous control genes) to predict the risk of distant recurrence for hormonereceptor-positive breast cancer between 5 to 10 years after diagnosis (prerequisites are that the patients have been 
treated with hormonal therapy and surgery, and are stage I or stage II lymph-node negative, or in stage II with one to three positive nodes). The test development started with a microarray discovery study and involved a multistage filtering, using consecutive applications of statistical tests and $\mathrm{CV}$ to propose a subset of candidate gene markers. ${ }^{54}$ The authors compared the reproducibility of classification scores obtained with these markers for three centroid-based prediction methods to ensure the robustness of the methodology. By further developing the approach into a more sensitive PCR-based test, and later into an assay using the NanoString nCounter Dx Analysis System, the predictive performance was improved in a stepwise fashion. The original discovery study was characterised by significantly larger sample sizes than the majority of reviewed biomarker studies, with a training set of 189 samples, test sets of 761 patients evaluated for prognosis, and 133 patients evaluated for prediction of pathologic complete response to treatment with taxane and anthracycline. These study design features in combination with multistage filtering and validation approaches, and improved measurement technology during the course of the study, may explain the successful progression of the PAM50 test to FDA clearance. The test has only three genes in common with the MammaPrint approach (KNTC2, MELK, ORC6L), which may be explained by the different technical and analytical approaches used, but a previous comparative evaluation concluded that the tests provide broadly equivalent risk information for females with oestrogen receptor (ER)-positive breast cancers. ${ }^{55}$

Among the LDTs for breast cancer prognosis, Oncotype $D X$ is a further test commonly used in clinical practice. ${ }^{86-59}$ The underlying gene signature consists of 16 cancer-associated genes and five reference genes, and is therefore often also referred to as '21-gene assay'. Its main application is to predict risk of recurrence in oestrogen-receptor positive tumours. The relevance of this prognostic tool for treatment selection may be explained by the strong association of the provided recurrence score with the probability of positive treatment response to chemotherapy. ${ }^{60}$ Oncotype $D X$ was developed using a consecutive refinement procedure, starting with the reverse transcription-polymerase chain reaction (RT-PCR) assessment of 250 candidate genes across 447 patients from three distinct studies to identify the 21-gene signature after multiple filtering steps. A recurrence score algorithm built using the signature as input was clinically validated on 668 independent patients. ${ }^{61}$ The selection of the 16 cancer-related genes included in the assay involved scoring the performance of the candidate features in all three studies and the consistency of the primer/probe performance in the assay. ${ }^{62}$ Thus, particular strengths of the development process for this LDT include the consideration of both technical robustness and statistical robustness of the assay across distinct cohorts. The Oncotype DX signature shares one gene with MammaPrint (SCUBE2), and nine genes with the Prosigna PAM50 test (BIRC5, CCNB1, MYBL2, MMP11, GRB7, ESR1, PGR,
$B C L, B A G 1)$. However, an independent clinical validation of Oncotype DX and the PAM50 signature for estimating the likelihood of distant recurrence in ER-positive, nodenegative, post-menopausal breast cancer patients treated with endocrine therapy suggested that the PAM50 signature provided more prognostic information than Oncotype $D X^{63}$

While the first validated omics biomarker signatures were developed for breast cancer, similar diagnostic and prognostic tools have followed for other cancer types. One of these is the Decipher Prostate Cancer Test, ${ }^{964-68}$ which differs from other omics-derived diagnostic tools by being provided together with a software platform and database, the Decipher Genomic Resource Information Database (GRID), that captures 1.4 million expression markers per patient to facilitate personalised care. The test itself uses 22 preselected RNAs to predict clinical metastasis and cancer-specific mortality for patients who have undergone radical prostatectomy. An initial discovery study by the Mayo Clinic (Rochester, Minnesota, USA) investigated a cohort of 545 such patients, split into a training $(\mathrm{n}=359)$ and a validation cohort $(n=186)$. Similar to other LDTs, the discovery started with a genome-wide profiling and used both statistical and ML analyses for filtering. First, t-tests were applied (reduction from 1.4 mil. to 18902 differentially expressed RNAs), then regularised logistic regression (reduction to 43 candidate markers), and finally a random forest-based feature selection (reduction to final set of 22 RNAs). Apart from testing the signature in the validation cohort, further external validations were performed in subsequent studies. ${ }^{94-68}$ Overall, distinctive strengths of the used approach include the improved interpretability of the test results through supporting analyses on the GRID platform, and the robustness of the discovery and validation approach, involving large sample sizes and several complementary statistical and ML assessments.

While most diagnostic tests in oncology have been designed for specific cancer types, a dedicated LDT has also been developed for cancers of unknown or uncertain diagnosis. The Cancer Type ID test by bioTheranostics distinguishes between 50 different tumour types using a 92-gene RT-PCR expression measurement signature. ${ }^{1569-71}$ This signature was derived from analyses of a microarray data collection covering 446 frozen tumour samples and 112 formalin-fixed, paraffin-embedded (FFPE) samples of both primary and metastatic tumours. Modelling steps involved k-nearest neighbour clustering and classification, and a genetic algorithm to explore the search space of possible feature subset selections. After successful CV (84\% accuracy) and external validation (82\% accuracy on 112 independent FFPE samples), the microarray-based signature was further developed to use more sensitive RT-PCR measurements. Testing the new approach on an independent validation set provided an increased accuracy (87\%). Distinctive characteristics of the development process that may have contributed to the positive validation include the efficient and extensive 
exploration of the search space of possible gene subset selections via a genetic algorithm, the large sample sizes used for discovery and validation, and the transfer of the assay from microarrays to the more sensitive RT-PCR platform.

The first omics-derived biomarker signatures addressed only the most frequent cancer types, but more recent applications in oncology focus on the diagnosis of less common malignancies, such as thyroid cancer. Typically, deciding whether a thyroid nodule is benign or cancerous is possible via a fine needle aspiration (FNA) biopsy, without requiring more complex measurements or analyses. However, while direct FNA-based diagnosis is feasible in most cases, indeterminate results can occur. ${ }^{72}$ To help prevent unnecessary surgeries for the corresponding patients, a molecular signature and LDT known as the Afirma Gene Expression Classifier (GEC) has been developed to discriminate benign from cancerous thyroid nodules. ${ }^{72-77}$ The original discovery study behind the GEC signature used mRNA expression analysis in 315 thyroid nodules, covering 178 retrospective surgical tissues and 137 prospectively collected FNA samples. Two ML classifiers were trained separately on surgical tissues and FNAs, assessing the test set performance on 48 independent, prospective FNA samples (50\% of which had indeterminate cytopathology). Discriminative features were selected using a linear modelling approach implemented in the software Limma, and a linear support vector machine was applied for model building and performance estimation via 30-fold CV. The successful CV results were confirmed on multiple distinct cohorts. ${ }^{72}$ 75-78 While the internal validation used in the initial study cannot address cohortspecific biases, the combined use of established feature selection and modelling approaches, and the subsequent external validation across multiple cohorts with large sample sizes may account for the successful translation of this signature.

Most omics-based diagnostic tests identified in our study rely purely on gene expression profiling data. However, more recently, first multiomics signatures for diagnostic purposes have been developed. One of the first LDTs that integrated information from both RNA and DNA sequencing was the FoundationOne Heme assay. ${ }^{14}{ }^{79-81}$ This assay aims to detect haematologic malignancies, sarcomas, paediatric malignancies or solid tumours (including among others leukaemias, myelodysplastic syndromes, myeloproliferative neoplasms, lymphomas, multiple myeloma, Ewing sarcoma, leiomyosarcoma and paediatric tumours). The test identifies four types of genomic alterations (base substitutions, insertions and deletions, copy number alterations, rearrangements) and reports microsatellite instability and tumour mutational burden to facilitate clinical decision making. This approach was originally developed and evaluated using reference samples of pooled cell lines in order to model the main characteristics that determine the test accuracy, including mutant allele frequency, indel length and amplitude of copy change. ${ }^{79}$ A first validation using 249 independent FFPE cancer samples, which had already been characterised by established assays, confirmed the accuracy of the test. External validation studies on independent cohorts corroborated the utility of the test for further diagnostic applications. ${ }^{14} 82$ The study results highlight the potential of integrating diverse biological data sources in order to obtain more robust and reliable predictions, a strategy that may be promising in particular for complex disorders that involve very heterogeneous phenotypes.

A common limitation of genomic profiling approaches for diagnostic testing is that most analyses have to be performed in centralised specialty laboratories, which limits a wider use and results in long waiting times. To address this shortcoming, the Elio Tissue Complete assay, an in vitro diagnostic test cleared in 2020 by the FDA for assessing somatic mutations and tumour mutation burden (TMB) in solid tumours, has been developed as an integrated DNA-to-report approach to enable a decentralised evaluation in all diagnostic labs with next generation sequencing (NGS) technology. ${ }^{83}$ The analytical performance of the test was assessed by comparing it with the FoundationOne test (see above) using a concordance analysis on 147 tumour specimens. It provided a positive percent agreement (PPA) above $95 \%$ for single nucleotide variants (SNVs) and insertions/deletions, and 80\%-83\% PPA for copy number alterations and gene translocations. ${ }^{83}$ The test has recently also been applied to investigate the response to immune checkpoint inhibitors (ICIs) in metastatic renal cell carcinoma, using a retrospective evaluation of SNVs, TMB, microsatellite status and genomic status of antigen presentation genes. ${ }^{84}$ While no correlation between treatment response and TMB was observed, one-third of patients with progressive disease following ICI therapy displayed loss of heterozygosity of major histocompatibility complex class I genes vs $6 \%$ of disease control patients, suggesting that loss of antigen presentation may restrict ICI response. ${ }^{84}$ In summary, the Elio Tissue Complete assay provides an example of how integrating NGS analyses with bioinformatics in a combined DNA-to-report approach could help to broaden the access to genomic diagnostics for both clinical and research applications.

\section{Non-cancer approved omics-derived diagnostic tests (four studies)}

While most clinically approved omics-derived diagnostic tests have been developed in the field of oncology, one of the first LDTs that received FDA clearance for a noncancer disease was the AlloMap Heart test. ${ }^{13}{ }^{85-87}$ It uses a gene expression signature of 11 target genes and 9 control genes in peripheral blood from heart transplant recipients to estimate the risk for acute cellular cardiac allograft rejection. The development process involved statistical analyses of leucocyte microarray profiling data from 285 samples, and subsequent RT-PCR validation and bioinformatics postprocessing. ${ }^{13}$ Prior knowledge from database and literature mining was included in the 
analysis by mapping the data to known alloimmune pathways. This allowed the researchers to narrow down 252 candidate marker genes. An RT-PCR validation on 145 samples confirmed 68 of these candidate genes, which distinguished rejection samples from quiescent samples according to a t-test $(p<0.01)$. Six genes were eliminated due to significant variation in gene expression with sample processing time. Next, the investigators averaged correlated gene expression levels to create robust metalevel features, called 'metagenes', and added 20 of these features as new variables. A linear discriminant analysis was applied, providing a prediction model using four individual genes and three metagenes, which aggregate information from 11 original genes. Finally, bootstrap validation procedures and external test set validations were performed to confirm the accuracy of this signature. Overall, distinctive aspects of the development approach for the AlloMap signature include the knowledge-based gene discovery, a comprehensive RT-PCR validation of candidate genes, and the robust bootstrap and external validation analyses.

The first clinically validated LDT for a cardiovascular indication derived from omics data was the Corus coronary artery disease (CAD) test, developed to identify CAD in stable non-diabetic patients. ${ }^{11}{ }^{88-91}$ In contrast to most other omics-based tests, Corus CAD is not a pure molecular signature test, but takes the clinical covariates gender and age into account. The initial discovery study used a retrospective microarray analysis of blood samples from 195 diabetic and non-diabetic patients from the Duke University CATHGEN registry. After ranking the studied genes by the statistical significance of group differences and prior biological knowledge on their disease relevance, 88 genes were selected for RT-PCR validation. Because diabetes status as a clinical covariate was significantly associated with the observed gene expression alterations, and the identified CAD-associated genes did not overlap between diabetic and non-diabetic patients, the authors decided to limit follow-up work to non-diabetic patients. In a prospective clinical trial, microarray profiling was conducted on blood samples from 198 patients, and topranked genes were further validated using RT-PCR for 640 blood samples. After multiple filtering steps, taking into account statistical significance in t-tests, biological relevance, gene correlation clustering and cell-type analyses, a final signature of 23 genes was derived, composed of 20 CAD-associated genes and 3 reference genes. ${ }^{92}$ To maximise the predictive performance, the final prediction algorithm was optimised to adjust for differences associated with age and gender. Compared with most other reviewed studies, the Corus CAD approach stands out by taking clinical covariates into account in the final prediction model, including an intermediate critical review and adjustment of the inclusion criteria (limiting the focus to nondiabetic patients), and integrating complementary filtering and validation analyses on large sample sizes.

For inflammatory diseases, a first omics-derived signature recently received approval for measuring rheumatoid arthritis (RA) inflammatory disease activity, the Vectra DA multibiomarker test. ${ }^{93-97}$ It uses blood serum samples and multispot 96-well immunoassay plates to assess serum concentrations of 12 protein biomarkers associated with the pathobiology of RA. The original Vectra DA score, which combines these measurements into a composite score between 1 and 100, was assessed via multivariate regression and displayed a high predictive power in estimating a standard RA score, the Disease Activity Score in 28 joints using the $\mathrm{C}$ reactive protein level (DAS28-CRP), in both seropositive (area under the receiver operating characteristic curve (AUC): 0.77, $\mathrm{p}<0.001$ ) and seronegative (AUC: $0.70, \mathrm{p}<0.001$ ) patients. ${ }^{97}$ This score was later adjusted for age, gender and adiposity (based on leptin concentration), and validated in two cohorts against DAS28-CRP as a prognostic test for radiographic progression during the next year. The results showed that the new adjusted score was the most accurate independent predictor of progression, with the rate of progression increasing from $<2 \%$ in the low ${ }^{1-29}$ adjusted score category to $16 \%$ in the high $^{45-100}$ category. ${ }^{95}$ Overall, the Vectra DA approach illustrates the utility of omicsbased biomarker signatures for prognostic applications in inflammatory disorders, and further highlights the benefit of integrating omics signatures with information from clinical covariates.

For neurodegenerative disorders, clinically approved diagnostic and prognostic omics-derived tests are still lacking. However, recently the Helix Genetic Health Risk App for Late-onset Alzheimer's Disease (AD) was cleared by the FDA for over-the-counter use. It detects clinically relevant variants in genomic DNA isolated from human saliva of individuals $\geq 18$ years in order to report and interpret genetic health risks, and evaluates the information of variants with established genome-wide significant associations to $\mathrm{AD}$. When tested on 99 human saliva samples, the accuracy was $100 \%$ with a lower $95 \%$ CI bound of $96.3 \% .^{98}$ The approach uses a whole exome sequencing (WES) constituent device, the Helix Laboratory Platform, ${ }^{99-101}$ as a qualitative in vitro diagnostics approach covering measurements for approximately 20k genes. The Helix Laboratory Platform has received FDA clearance through a new regulatory approval pathway established by the FDA for WES devices (Regulation 21 CFR 866.6000). Due to the generic applicability of the WES profiling assay used by this platform, called Exome+, the assay has also been applied to find statistically significant gene-based associations for several other phenotypes in large-scale cohort studies $^{99}$ and to identify carriers of autosomal dominant diseases by population-based genetic screening. ${ }^{101}$ Thus, the Helix Laboratory Platform provides a first example for a new approval pathway for omics-based diagnostic tests, in which a clinically approved genomic testing device is not anymore linked to a single diagnostic application or a specific disease type. Instead, the market authorisation for diagnostic tests is obtained separately from the device and facilitated and accelerated by the prior approval of the constituent measurement device. For the future 


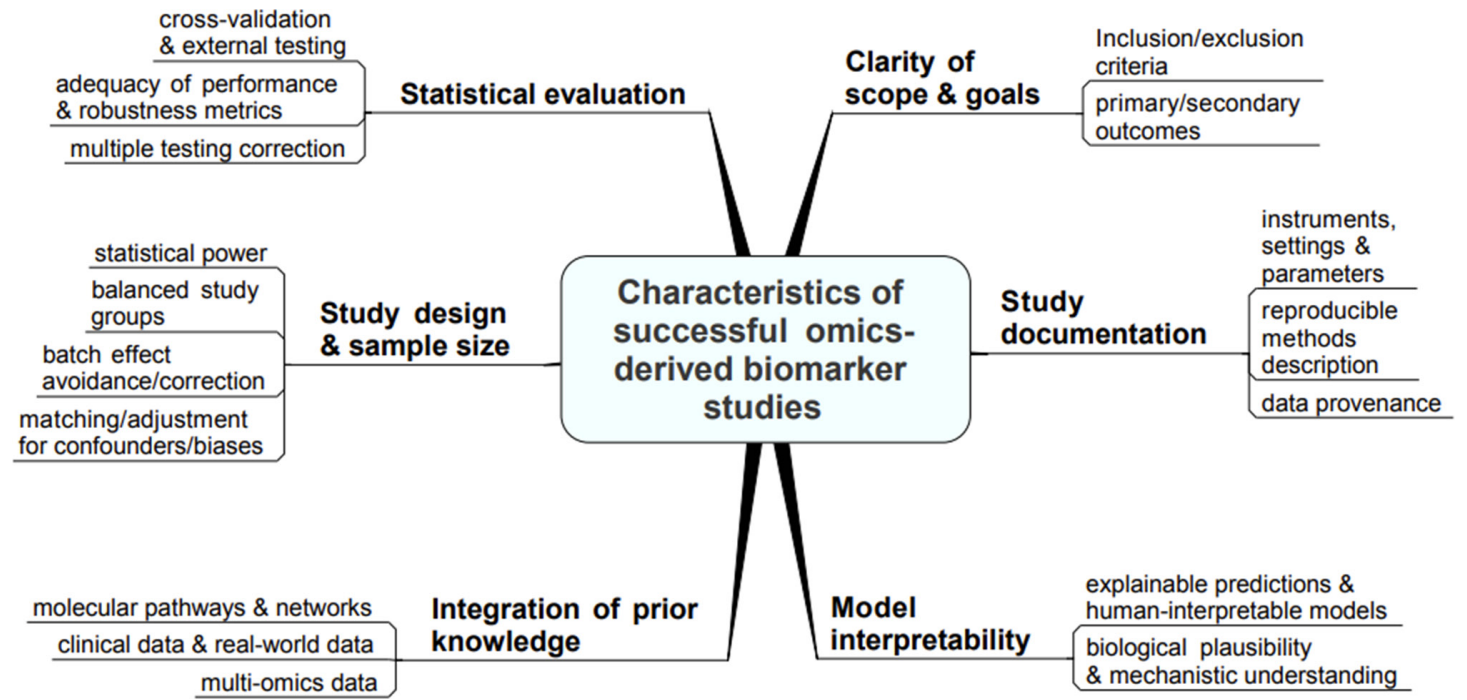

Figure 6 Characteristics of successful omics-based studies. Six main categories of design and implementation aspects that characterise successful omics-based biomarker development studies were identified (starting from the centre left in the figure and proceeding clockwise): (1) adequacy of the study design and sample size selection; (2) rigour and robustness of the statistical evaluation; (3) clarity of scope and goals; (4) completeness and reproducibility of the study documentation; (5) interpretability and biological plausibility of the created predictive models; (6) integration of prior biological knowledge into the model building and validation procedures.

development of omics-derived biomarker signatures, this may allow researchers to focus on demonstrating the clinical utility of a new signature, while the analytical validity of the underlying testing device has already been established previously.

\section{DISCUSSION}

\section{Statement of principal findings}

The scoping review of articles on patient stratification using omics data revealed common limitations in the study design for many published biomarker development projects, such as insufficient and imbalanced sample sizes per study group and inadequate validation methods, but also identified multiple studies that have led to validated diagnostic and prognostic tests. These success stories were investigated in more detail to identify common characteristics in the study design, discovery and validation methods, which may have supported the clinical translation of the initial findings. Figure 6 outlines key shared aspects that are possible determinants of the study success and could help to guide future biomarker investigations. In particular, they cover the following main features:

1. A sample size selection, study group and replicate design that provides adequate statistical power for the ML analyses.

2. The application of robust statistical filtering and evaluation schemes (including multiple layers of statistical and ML-based feature selection, combined statistical and biological filters, robust validation schemes that involve multiple CV, bootstrapping and external validation analyses, using multiple suitable and complementary performance metrics, and providing information on the statistical variation and confidence intervals for the performance estimates, see figure 7 for an overview of recommended generic steps for robust model building and evaluation).

3. Clarity of the study scope and goals (involving clear inclusion and exclusion criteria, primary and secondary outcomes, and decision processes to make necessary adjustments due to new knowledge gained during the project, such as the adjusted inclusion criteria in the Corus CAD study and the progression from nontargeted microarray technology to higher-sensitivity RT-PCR in the case of the Prosigna test and the Cancer Type ID test).

4. Completeness and reproducibility of the study documentation (covering details on used instruments, parameters and settings, reproducible methods descriptions and information on data provenance).

5 . Interpretability and biological plausibility of the created predictive models (including explainable and justifiable predictions, human-interpretable model descriptions, and biologically plausible models that agree with the current mechanistic understanding of the studied disorder).

6. Integration of prior biological knowledge into the predictive feature selection, model building and validation procedures (eg, using public data on disease-associated molecular pathways and networks; complementary clinical and real-world data, and relevant multiomics data).

\section{Strengths and limitations}

The majority of methodological recommendations derived from the study relate to the early planning and study design for biomarker discovery projects, involving considerations associated with the choice of the study 
group, sampling and blocking design, the measurement technology, and the input and output variables. ${ }^{16} 17$ These recommendations are therefore mainly applicable to prospective studies. For retrospective biomarker investigations of already collected data, the suggestions derived from the review are limited to guidance on improving analysis workflows, for example, for filtering and evaluation analyses, the integration of prior knowledge from multiomics data and public annotation databases, and the choice of robust and interpretable modelling approaches for the generation of biologically plausible and reproducible prediction models. While the focus of the review on studies that have already led to validated biomarker models and that fulfil minimum requirements for sample size and statistical model assessment helps to ensure the quality of the selected articles, no further quality evaluation was performed. The reader should also note the generic limitations of ML methods which can affect all biomarker studies: These include the necessity for a representative coverage of the relevant outcomes in the training and validation groups, a sufficiently comprehensive and sensitive coverage of informative predictor variables in the data for the outcomes of interest, which may not be achievable for omics data from tissues and body fluids with limited disease relevance or measurement sensitivity, and a sufficient data quality in terms of the influence of systematic biases and noise. Moreover, for multiomics biomarker analyses, in addition to adequate pre-processing and ML approaches, suitable strategies and methods for the integration of diverse omics data are also needed. These multiomics data integration strategies were not within the scope of the present review, but have been reviewed in previous publications. ${ }^{102-104}$ Finally, more recent methodological developments in the ML and CV analysis of omics data, such as meta-learning ${ }^{105}$ and bolstered $\mathrm{CV},{ }^{106}$ have only limited coverage among the articles that passed the eligibility criteria, and will therefore require further dedicated study in the future.

\section{Discussing important differences in results}

Previous reviews of ML approaches using omics data for patient stratification have focused on domain-specific analyses for specific types of diseases, or specific types of ML methodologies. ${ }^{107-115}$ By contrast, this scoping review focuses on disease-agnostic workflows with generic applicability across complex human disorders involving multifactorial molecular alterations. The coverage of statistical and ML approaches for stratification does not aim to provide a detailed discussion of specific algorithms, statistical methods or scoring metrics, but rather at identifying key determinants of success for generic analysis and validation workflows in biomedical stratification studies. Therefore, the results describe general workflow characteristics that distinguish omics biomarker studies with clinical translation from other studies, and cover associated disease-agnostic recommendations for future studies, whereas method recommendations specific to particular disease types or ML analysis types are covered elsewhere in domain-specific reviews. ${ }^{107-115}$

\section{Meaning of the study: implications for clinicians and policy- makers}

The previous clinical translation successes in omicsbased biomarker development reviewed in this study, which have mostly been achieved in the field of oncology, highlight the potential for developing similar biomarker signatures for further disease indications. In contrast to conventional statistical biomarker discovery approaches, which focus on identifying single-molecule markers, systems-level analysis of omics data using multivariate ML approaches can identify multifactorial signatures which are robust against noise in individual gene or protein measurements, and more biologically insightful by reflecting disease-associated cellular process alterations in a more comprehensive fashion.

This scoping review has identified common characteristics of omics studies which have led to clinically validated diagnostic and prognostic tests. Thus, the conclusions drawn on recommended practices for

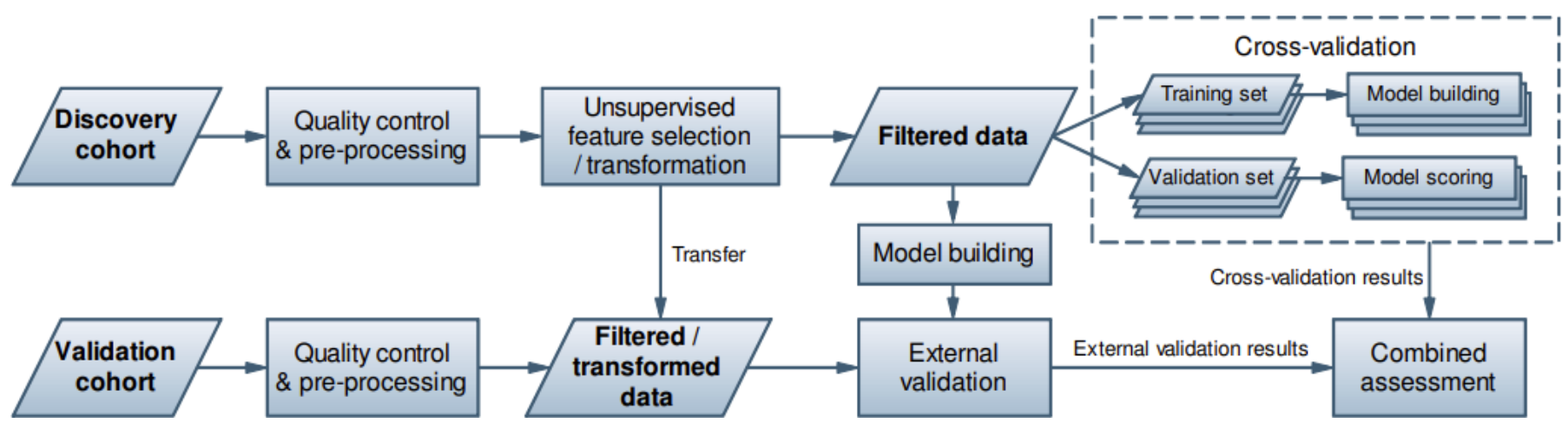

Figure 7 Recommended generic workflow for biomarker development using machine learning analysis of omics data. The machine learning analysis of omics data for biomarker discovery and validation should ideally involve dedicated quality control and preprocessing analyses, a dimension reduction using unsupervised feature selection (eg, a variance filtering) or data transformation approaches (eg, using a principal components analysis), a cross-validation on the discovery cohort, and an external validation on a distinct validation cohort. 
sample size selection, biological data filtering and ML, and the implementation of adequate validation schemes may help to guide clinical researchers on study design choices and the selection of analysis methodologies. Additionally, the scoping review results can help to raise awareness of common pitfalls, such as issues associated with batch effects, biases, confounding factors, lack of statistical power and multiple hypothesis testing, and thus contribute to preventing these failure causes in biomarker development. For policy-makers and funding bodies, findings on the distinctive characteristics of studies with successful clinical biomarker translation, for example, concerning the specific requirements for robust CV and external result validation methods, may provide relevant information for the design of public and private funding schemes for biomedical research. Risks in funded research projects may be addressed upfront through appropriate guidelines and regulations for the study design and validation (eg, recommendations on power calculations and specific validation and documentation requirements). Finally, the scoping review results can guide clinicians involved in biomarker discovery on how to make better use of available public knowledge and data sources, for example, cellular pathway and molecular interaction databases, that may allow them to exploit prior knowledge effectively, and create more robust and interpretable biomarker models.

\section{Unanswered questions and future research}

Since the recommendations and guidelines identified from the reviewed articles are mostly derived from established biomarker discovery and validation approaches, new methodologies and upcoming trends could only be covered to a limited extent and may lead to changed recommendations in the future. In particular, in the reviewed patient stratification studies, some of more recently introduced ML concepts (eg, transfer learning, distance metric learning, semisupervised learning, structured ML, meta learning, multiview learning and generative models), data processing techniques (eg, new dimension reduction approaches, outlier removal methods, data augmentation techniques) and model validation methods (eg, bootstrapping or bolstered CV, uncertainty quantification), are still underrepresented among the eligible studies reviewed, and may provide suitable topics for follow-up research.

Overall, while the currently available literature on validated stratification biomarkers already provides ample information on common pitfalls and established practices, the development of widely accepted standard guidelines on methodologies for omics biomarker discovery will require further knowledge exchange and deliberation among stakeholders in the field. In particular, integration of domain-specific expertise in discussions involving clinicians, experimental and data scientists, and regulatory and legal experts is required as a follow-up effort to derive comprehensive methodological guidelines for future biomarker development.
Correction notice This article has been corrected since it was first published. The author byline section has been updated.

AcknowledgmentsThe authors thank Vanna Pistotti for her assistance with search strategy development and conduction.

Collaborators PERMIT group: 1. Antonio L. Andreu 2. Florence Bietrix 3. Florie Brion Bouvier 4. Montserrat Carmona Rodriguez 5. Maria del Mar Polo-de Santos 6. Maddalena Fratelli 7. Rainer Girgenrath 8. Alexander Grundmann 9. Josep Maria Haro 10. Frank Hulstaert 11. Iñaki Imaz-Iglesia 12. Setefilla Luengo Matos 13. Emmet McCormack 14. Albert Sanchez Niubo 15. Emanuela Oldoni 16. Raphael Porcher 17. Vibeke Fosse 18. Luis M. Sánchez-Gómez 19. Lorena San Miguel 20. Cecilia Superchi 21. Teresa Torres 22. Anna Monistrol Mula

Contributors Study conception and design: EG and AR. Methodology: CG and RB. Data collection and analysis: EG and AR. Original draft preparation: EG. Review and editing: AR, EG, PG, CG, JD and RB. Project supervision: PG. Funding acquisition: JD. Responsible for the overall content as guarantor: EG. All authors have read and revised the manuscript and approved the final version. The members of the PERMIT group were involved in the preparation or revision of the joint protocol of the four scoping reviews of the PERMIT series, attended the joint workshop (consultation exercise) and are coauthors of the other scoping reviews of the PERMIT series.

This project has received funding from the European Union's Horizon 2020 research and innovation programme under grant agreement No. 874825 .

Map disclaimer The inclusion of any map (including the depiction of any boundaries therein), or of any geographic or locational reference, does not imply the expression of any opinion whatsoever on the part of BMJ concerning the legal status of any country, territory, jurisdiction or area or of its authorities. Any such expression remains solely that of the relevant source and is not endorsed by BMJ. Maps are provided without any warranty of any kind, either express or implied.

Competing interests None declared

Patient consent for publication This study did not require consent from patients, because it does not use individual data.

Ethics approval This study was based entirely on a scoping review of relevant published literature and did not require an ethics approval.

Provenance and peer review Not commissioned; externally peer reviewed.

Data availability statement The study protocol was published on the online platform Zenodo.$^{19}$ Copies of searches and data extraction sheets will be made publicly available on Zenodo as part of the database collection for all scoping reviews conducted in the PERMIT project.

Supplemental material This content has been supplied by the author(s). It has not been vetted by BMJ Publishing Group Limited (BMJ) and may not have been peer-reviewed. Any opinions or recommendations discussed are solely those of the author(s) and are not endorsed by BMJ. BMJ disclaims all liability and responsibility arising from any reliance placed on the content. Where the content includes any translated material, BMJ does not warrant the accuracy and reliability of the translations (including but not limited to local regulations, clinical guidelines, terminology, drug names and drug dosages), and is not responsible for any error and/or omissions arising from translation and adaptation or otherwise.

Open access This is an open access article distributed in accordance with the Creative Commons Attribution Non Commercial (CC BY-NC 4.0) license, which permits others to distribute, remix, adapt, build upon this work non-commercially, and license their derivative works on different terms, provided the original work is properly cited, appropriate credit is given, any changes made indicated, and the use is non-commercial. See: http://creativecommons.org/licenses/by-nc/4.0/.

\section{ORCID iDs}

Enrico Glaab http://orcid.org/0000-0003-3977-7469

Armin Rauschenberger http://orcid.org/0000-0001-6498-4801

Rita Banzi http://orcid.org/0000-0002-2211-3300

Chiara Gerardi http://orcid.org/0000-0002-2459-4769

Jacques Demotes http://orcid.org/0000-0002-0807-0746

\section{REFERENCES}

1 Reel PS, Reel S, Pearson E, et al. Using machine learning approaches for multi-OMICS data analysis: a review. Biotechnol Adv 2021;49.

2 Goecks J, Jalili V, Heiser LM, et al. How machine learning will transform biomedicine. Cell 2020;181:92-101. 
3 Jiang $\mathrm{Y}$, Wang M. Personalized medicine in oncology: tailoring the right drug to the right patient. Biomarkers in Medicine. 2010.

4 Hopp WJ, Li J, Wang G. Big data and the precision medicine revolution. Prod Oper Manag 2018;27:1647-64.

5 Glaab E. Using prior knowledge from cellular pathways and molecular networks for diagnostic specimen classification. Brief Bioinform 2016

6 Cardoso F, van't Veer LJ, Bogaerts J, et al. 70-Gene signature as an aid to treatment decisions in early-stage breast cancer. $N$ Engl J Med 2016;375:717-29.

7 Bachleitner-Hofmann T, Simon I, Salazar R, et al. Development and validation of a robust molecular diagnostic test (COLOPRINT) for predicting outcome in stage II colon cancer patients. Ann Oncol. 2012.

8 Knezevic D, Goddard AD, Natraj N, et al. Analytical validation of the Oncotype DX prostate cancer assay - a clinical RT-PCR assay optimized for prostate needle biopsies. BMC Genomics 2013;14.

9 Torres A, Alshalalfa M, Tomlins SA, et al. Comprehensive determination of prostate tumor Ets gene status in clinical samples using the CLIA decipher assay. J Mol Diagnostics 2017;19:475-84.

10 Angell TE, Babiarz J, Barth N, et al. Clinical validation of the AFIRMA genomic sequencing BRAF V600E classifier. Thyroid [Internet] 2017;27:A50 http://www.embase.com/search/results? subaction=viewrecord\&from $=$ export\&id $=\mathrm{L} 624116485$

11 Ladapo JA, Budoff MJ, Sharp D, et al. Utility of a precision medicine test in elderly adults with symptoms suggestive of coronary artery disease. J Am Geriatr Soc 2018;66:309-15.

12 Tabari E, Lovejoy AF, Lin H, et al. Molecular characteristics and disease burden metrics determined by next-generation sequencing on circulating tumor DNA correlate with progression free survival in previously untreated diffuse large B-cell lymphoma. Blood [Internet] 2019;134. [Epub ahead of print: Available from].

13 Deng MC. The AlloMapTM genomic biomarker story: 10 years after Clin Transplant. 2017;31.

$14 \mathrm{He}$ J, Abdel-Wahab O, Nahas MK, et al. Integrated genomic DNA RNA profiling of hematologic malignancies in the clinical setting. Blood 2016;127:3004-14.

15 XJ M, Patel R, Wang X, et al. Molecular classification of human cancers using a 92-gene real-time quantitative polymerase chain reaction assay. Arch Pathol Lab Med 2006;130:465-73.

16 McShane LM, Cavenagh MM, Lively TG, et al. Criteria for the use of OMICs-based predictors in clinical trials.. Nature 2013.

17 Moons KGM, Altman DG, Reitsma JB, et al. Transparent reporting of a multivariable prediction model for individual prognosis or diagnosis (TRIPOD): explanation and elaboration. Ann Intern Med 2015:162:W1-73.

18 Banzi R, Gerardi C, Fratelli M, et al. Web-page for the Personalized Medicine Trials (PERMIT) project [Internet]., 2020. Available: https:// permit-eu.org [Accessed cited 2021 Aug 2].

19 Banzi R, Gerardi C, Fratelli M, et al. Methodological approaches for personalised medicine: protocol for a series of scoping reviews [Internet]. 10.5281/zenodo.3770937. Available: https://zenodo.org/ record/3770937

20 Perlis RH. Translating biomarkers to clinical practice. Mol Psychiatry 2011;16:1076-87.

21 Graaf G, Postmus D, Westerink J, et al. The early economic evaluation of novel biomarkers to accelerate their translation into clinical applications. Cost Eff Resour Alloc 2018;16.

22 Drucker E, Krapfenbauer K. Pitfalls and limitations in translation from biomarker discovery to clinical utility in predictive and personalised medicine. EPMA Journal. 2013;4.

23 Williams JK, Anderson CM. Omics research ethics considerations. Nurs Outlook. 2018.

24 Vähäkangas K. Research ethics in the post-genomic era. Environmental and Molecular Mutagenesis 2013.

25 Olson RS, La Cava W, Orzechowski P, et al. PMLB: a large benchmark suite for machine learning evaluation and comparison. BioData Min. 2017.

26 Rappoport N, Shamir R. Multi-Omic and multi-view clustering algorithms: review and cancer benchmark. Nucleic Acids Res [Internet] 2018;46:10546-62 http://www.embase.com/search/ results?subaction=viewrecord\&from=export\&id=L626271229

27 Herrmann M, Probst P, Hornung R, et al. Large-Scale benchmark study of survival prediction methods using multi-omics data. Brief Bioinform 2021.

28 Peters MDJ, Godfrey CM, Khalil H, et al. Guidance for conducting systematic scoping reviews. Int J Evid Based Healthc 2015;13:141-6.

29 Pham MT, Rajić A, Greig JD, et al. A scoping review of scoping reviews: advancing the approach and enhancing the consistency. Res Synth Methods 2014;5:371-85.
30 Munn Z, Peters MDJ, Stern C, et al. Systematic review or scoping review? guidance for authors when choosing between a systematic or scoping review approach. BMC Med Res Methodol 2018;18:143.

31 Tricco AC, Lillie E, Zarin W, et al. PRISMA extension for scoping reviews (PRISMA-ScR): checklist and explanation. Ann Intern Med 2018;169:467-73.

32 Van't Veer LJ, Dai H, Van de Vijver MJ, et al. Gene expression profiling predicts clinical outcome of breast cancer. Nature 2002;415:530-6.

33 Ouzzani M, Hammady H, Fedorowicz Z, et al. Rayyan-a web and mobile APP for systematic reviews. Syst Rev 2016;5:210.

34 Glaab E, Rauschenberger A, Banzi R. Data from: Selected articles from the scoping review of biomarker discovery studies for the EU project on "Personalised Medicine Trials" (PERMIT). Zenodo, November 4, 2021. https://doi.org/10.5281/zenodo.5646467

35 Rahman M, Fukui T. Biomedical research productivity: factors across the countries. Int J Technol Assess Health Care 2003;19:249-52.

36 Hwang TJ, Kesselheim AS, Vokinger KN. Lifecycle regulation of artificial Intelligence- and machine Learning-Based software devices in medicine. JAMA 2019;322:2285-6.

37 FDA Center for Devices and Radiological Health. Web-page on Nucleic Acid Based Tests by the Food and Drug Administration (FDA) [Internet]. 2021 [cited 2021 Aug 2]. Available: https://www.fda. gov/medical-devices/vitro-diagnostics/nucleic-acid-based-tests

38 Wittner BS, Sgroi DC, Ryan PD, et al. Analysis of the MammaPrint breast cancer assay in a predominantly postmenopausal cohort. Clin Cancer Res 2008;14:2988-93.

39 Slodkowska EA, Ross JS. MammaPrint ${ }^{\mathrm{TM}} 70$-gene signature: Another milestone in personalized medical care for breast cancer patients. Expert Rev Mol Diagn 2009;9:417-22.

40 Sapino A, Roepman P, Linn SC, et al. Mammaprint molecular diagnostics on formalin-fixed, paraffin-embedded tissue. J Mol Diagnostics 2014;16:190-7.

41 Mook S, Knauer M, Bueno-De-Mesquita JM, et al. Metastatic potential of T1 breast cancer can be predicted by the 70-gene MammaPrint signature. Ann Surg Oncol 2010;17:1406-13.

42 Maak M, Simon I, Nitsche U, et al. Independent validation of a prognostic genomic signature (ColoPrint) for patients with stage II colon cancer. Ann Surg 2013;257:1053-8.

43 Kopetz S, Tabernero J, Rosenberg R, et al. Genomic classifier ColoPrint predicts recurrence in stage II colorectal cancer patients more accurately than clinical factors. Oncologist 2015;20:127-33.

44 Tan IB, Tan P. Genetics: an 18-gene signature (ColoPrint ${ }^{\circledR}$ ) for colon cancer prognosis. Nat Rev Clin Oncol 2011;8:131-3.

45 Rosenberg R, Maak M, Simon I, et al. Independent validation of a prognostic genomic profile (ColoPrint) for stage II colon cancer (CC) patients. J Clin Oncol 2011;29:358

46 Salazar R, de Waard JW, Glimelius B, et al. The PARSC trial, a prospective study for the assessment of recurrence risk in stage II colon cancer (CC) patients using ColoPrint. J Clin Oncol 2012;30:678

47 Tabernero J, Moreno V, Rosenberg R, et al. Clinical and technical validation of a genomic classifier (ColoPrint) for predicting outcome of patients with stage II colon cancer. J Clin Oncol 2012;30:384

48 Bachleitner-Hofmann T, Simon I, Salazar R, et al. Development and validation of a robust molecular diagnostic test (COLOPRINT) for predicting outcome in stage II colon cancer patients. Ann Oncol 2012;23:ix179.

49 Nielsen T, Wallden B, Schaper C, et al. Analytical validation of the PAM50-based Prosigna breast cancer prognostic gene signature assay and nCounter analysis system using formalin-fixed paraffinembedded breast tumor specimens. BMC Cancer 2014;14.

50 Wallden B, Storhoff J, Nielsen T, et al. Development and verification of the PAM50-based Prosigna breast cancer gene signature assay. BMC Med Genomics 2015;8.

51 Alvarado MD, Prasad C, Rothney M, et al. A prospective comparison of the 21-gene recurrence score and the PAM50-Based Prosigna in estrogen receptor-positive early-stage breast cancer. Adv Ther 2015;32:1237-47.

52 Jensen MB, Lænkholm AV, Nielsen TO, et al. The Prosigna gene expression assay and responsiveness to adjuvant cyclophosphamide-based chemotherapy in premenopausal highrisk patients with breast cancer. Breast Cancer Res 2018;20.

53 Hequet D, Callens C, Gentien D, et al. Prospective, multicenter French study evaluating the clinical impact of the breast cancer intrinsic Subtype-Prosigna ${ }^{\circledR}$ test in the management of early-stage breast cancers. PLoS One 2017;12.

54 Parker JS, Mullins M, Cheang MCU, et al. Supervised risk predictor of breast cancer based on intrinsic subtypes. J Clin Oncol 2009:27:1160-7. 
55 Bartlett JMS, Bayani J, Marshall A, et al. Comparing breast cancer multiparameter tests in the optima prelim trial: no test is more equal than the others. J Nat/ Cancer Inst 2016;108.

56 Kelly CM, Krishnamurthy S, Bianchini G, et al. Utility of Oncotype DX risk estimates in clinically intermediate risk hormone receptorpositive, HER2-normal, grade II, lymph node-negative breast cancers. Cancer 2010;116:5161-7.

57 SS L, Mumby PB, Norton J, et al. Prospective multicenter study of the impact of the 21-gene recurrence score assay on medical oncologist and patient adjuvant breast cancer treatment selection. $J$ Clin Oncol 2010;28:1671-6.

58 Carlson JJ, Roth JA. The impact of the Oncotype DX breast cancer assay in clinical practice: a systematic review and meta-analysis. Breast Cancer Res Treat 2013;141:13-22.

59 Thakur SS, Li H, Chan AMY, et al. The use of automated Ki67 analysis to predict Oncotype DX risk-of-recurrence categories in early-stage breast cancer. PLoS One 2018/01/06. 2018;13:e0188983.

60 Pease AM, Riba LA, Gruner RA, et al. Oncotype DX $®$ recurrence score as a predictor of response to neoadjuvant chemotherapy. Ann Surg Oncol. 2019

61 Gianni L, Zambetti M, Clark K, et al. Gene expression profiles in paraffin-embedded core biopsy tissue predict response to chemotherapy in women with locally advanced breast cancer. J Clin Oncol 2005;23:7265-77.

62 Paik S, Shak S, Tang G, et al. A multigene assay to predict recurrence of tamoxifen-treated, node-negative breast cancer. $N$ Engl J Med 2004:351:2817-26.

63 Dowsett M, Sestak I, Lopez-Knowles E, et al. Comparison of PAM50 risk of recurrence score with Oncotype DX and IHC4 for predicting risk of distant recurrence after endocrine therapy. J Clin Oncol 2013;31:2783-90.

64 Marrone M, Potosky AL, Penson D, et al. A 22 gene-expression assay, decipher ${ }^{\circledR}$ (GenomeDx biosciences) to predict five-year risk of metastatic prostate cancer in men treated with radical prostatectomy. PLoS Curr. 2015;7:(EVIDENCEONGENOMI CTESTS).

65 Nguyen PL, Haddad Z, LLC L, et al. Et al. evaluation of the decipher prostate cancer classifier to predict metastasis and disease-specific mortality from genomic analysis of diagnostic prostate needle biopsy specimens. J Clin Oncol 2017;35:4

66 Magi-Galluzzi C, Yousefi K, et al, Lam LLC,. Et al. validation of the decipher prostate cancer classifier for predicting 10-year postoperative metastasis from analysis of diagnostic needle biopsy specimens. J Clin Oncol 2016;34:59

67 Dalela D, Löppenberg B, Sood A, et al. Contemporary role of the Decipher ${ }^{\circledR}$ test in prostate cancer management: current practice and future perspectives. Rev Urol 2016;18:1-9.

68 Klein EA, Haddad Z, et al, Lam LLC,. Et al. decipher genomic classifier measured on prostate biopsy predicts metastasis risk. Urology 2016;90:148-52.

69 Weiss LM, Chu P, Schroeder BE, et al. Blinded comparator study of immunohistochemical analysis versus a 92-gene cancer classifier in the diagnosis of the primary site in metastatic tumors. $J \mathrm{Mol}$ Diagnostics 2013;15:263-9.

70 Greco FA, Spigel DR, Yardley DA, et al. Molecular profiling in unknown primary cancer: accuracy of tissue of origin prediction. Oncologist 2010;15:500-6.

71 Hainsworth JD, Rubin MS, Spigel DR, et al. Molecular gene expression profiling to predict the tissue of origin and direct sitespecific therapy in patients with carcinoma of unknown primary site: a prospective trial of the Sarah Cannon research Institute. J Clin Oncol 2013;31:217-23.

72 Harrison G, Sosa JA, Jiang X. Evaluation of the Afirma gene expression classifier in repeat indeterminate thyroid nodules. Arch Pathol Lab Med 2017;141:985-9.

73 Chudova D, Wilde Jl, Wang ET, et al. Molecular classification of thyroid nodules using high-dimensionality genomic data. J Clin Endocrinol Metab 2010;95:5296-304.

$74 \mathrm{Kim} \mathrm{Ml}$, Alexander EK. Diagnostic use of molecular markers in the evaluation of thyroid nodules. Endocr Pract 2012;18:796-802.

75 Ali SZ, Fish SA, Lanman R. Use of the Afirma ${ }^{\circledR}$ gene expression classifier for preoperative identification of benign thyroid nodules with indeterminate fine needle aspiration cytopathology. PLoS Currents. 2013:1-7.

76 Mclver B, Castro MR, Morris JC, et al. An independent study of a gene expression classifier (Afirma) in the evaluation of cytologically indeterminate thyroid nodules. J Clin Endocrinol Metab 2014;99:4069-77

77 Lastra RR, Pramick MR, Crammer CJ, et al. Implications of a suspicious Afirma test result in thyroid fine-needle aspiration cytology: an institutional experience. Cancer Cytopathol 2014:122:737-44

78 Kim JY, Park SC, Lee JK, et al. Novel antibacterial activity of $\beta 2-$ microglobulin in human amniotic fluid. PLoS One 2012.

79 Frampton GM, Fichtenholtz A, Otto GA, et al. Development and validation of a clinical cancer genomic profiling test based on massively parallel DNA sequencing. Nat Biotechnol 2013;31:1023-31.

80 Wang K, Sanchez-Martin M, Wang X, et al. Patient-Derived xenotransplants can recapitulate the genetic driver landscape of acute leukemias. Leukemia 2017;31:151-8.

81 Tarlock K, He J, Zhong S, et al. Distinct age-associated genomic profiles in acute myeloid leukemia (AML) using FoundationOne heme. J Clin Oncol 2016;34:7041

82 Lieber DS, Kennedy MR, Johnson DB, et al. Abstract B16: validation and clinical feasibility of a Foundation medicine assay to identify immunotherapy response potential through tumor mutational burden (TMB). in 2017

83 Lee Deak K, Jackson JB, Valkenburg KC, et al. Next-Generation sequencing concordance analysis of comprehensive solid tumor profiling between a centralized specialty laboratory and the decentralized PGdx Elio tissue complete Kitted solution. J Mol Diagnostics [Internet] 2021 Julin press. [Epub ahead of print: Available from] https://linkinghub.elsevier.com/retrieve/pii/ S1525157821002105

84 Labriola MK, Zhu J, Gupta R, et al. Characterization of tumor mutation burden, PD-L1 and DNA repair genes to assess relationship to immune checkpoint inhibitors response in metastatic renal cell carcinoma. J Immunother Cancer [Internet]. 2020;8. [Epub ahead of print: Available from] https://jitc.bmj.com/lookup/doi/

85 Yamani MH, Taylor DO, Rodriguez ER, et al. Transplant vasculopathy is associated with increased AlloMap gene expression score. J Hear Lung Transplant 2007;26:403-6.

86 Yamani MH, Taylor DO, Haire C, et al. Post-Transplant ischemic injury is associated with up-regulated AlloMap gene expression. Clin Transplant 2007;21:523-5.

87 Kobashigawa J, Patel J, Azarbal B, et al. Randomized pilot trial of gene expression profiling versus heart biopsy in the first year after heart transplant: early invasive monitoring attenuation through gene expression trial. Circ Hear Fail 2015;8:557-64.

88 Wingrove JA, Daniels SE, Sehnert AJ, et al. Correlation of peripheral-blood gene expression with the extent of coronary artery stenosis. Circ Cardiovasc Genet 2008;1:31-8.

89 Rosenberg S, Dehais C, Ducray F. OS11.3 machine learning for better prognostic stratification and driver genes identification in 1p/19q-codeleted grade III gliomas. Neuro Oncol [Internet]. 2017;19. [Epub ahead of print: Available from]

90 Vargas J, Lima JAC, Kraus WE, et al. Use of the Corus ${ }^{\circledR}$ CAD gene expression test for assessment of obstructive coronary artery disease likelihood in symptomatic non-diabetic patients. PLoS Currents. 2013

91 Elashoff MR, Wingrove JA, Beineke P, et al. Development of a blood-based gene expression algorithm for assessment of obstructive coronary artery disease in non-diabetic patients. BMC Med Genomics. 2011;4.

92 Rosenberg S, Elashoff MR, Beineke P, et al. Multicenter validation of the diagnostic accuracy of a blood-based gene expression test for assessing obstructive coronary artery disease in nondiabetic patients. Ann Intern Med 2010;153:425-34.

93 Brahe $\mathrm{CH}$, Østergaard M, Johansen JS, et al. Predictive value of a multi-biomarker disease activity score for clinical remission and radiographic progression in patients with early rheumatoid arthritis: a post-hoc study of the opera trial. Scand J Rheumatol. 2019.

94 Chernoff D, Scott Eastman P, Hwang CC, et al. Determination of the minimally important difference (mid) in multi-biomarker disease activity (MBDA) test scores: impact of diurnal and daily biomarker variation patterns on MBDA scores. Clin Rheumatol 2019:38:437-45.

95 Curtis JR, Weinblatt ME, Shadick NA, et al. Validation of the adjusted multi-biomarker disease activity score as a prognostic test for radiographic progression in rheumatoid arthritis: a combined analysis of multiple studies. Arthritis Res Ther. 2021.

96 Curtis JR, Xie F, Yang S. Uptake and clinical utility of Multibiomarker disease activity testing in the United States. J Rheumatol [Internet] 2019;46. [Epub ahead of print: Available from] http://www.ncbi.nlm. nih.gov/pubmed/30442830

97 Curtis JR, Van Der Helm-Van Mil AH, Knevel R, et al. Validation of a novel multibiomarker test to assess rheumatoid arthritis disease activity. Arthritis Care Res 2012;64:1794-803.

98 Food and Drug Administration. Helix Genetic Health Risk App For Late-Onset Alzheimer's Disease - FDA Review Decision Summary., 
2020. Available: https://www.accessdata.fda.gov/scripts/cdrh/ cfdocs/cfpmn/pmn.cfm?ID=K192073

99 Cirulli ET, White S, Read RW, et al. Genome-Wide rare variant analysis for thousands of phenotypes in over 70,000 exomes from two cohorts. Nat Commun. 2020.

100 JT L, Ferber M, Hagenkord J, et al. Evaluation for genetic disorders in the absence of a clinical indication for testing: elective genomic testing. Journal of Molecular Diagnostics. 2019.

101 Grzymski JJ, Elhanan G, Morales Rosado JA, et al. Population genetic screening efficiently identifies carriers of autosomal dominant diseases. Nat Med. 2020.

102 Huang S, Chaudhary K, Garmire LX. More is better: recent progress in multi-omics data integration methods. Front Genet 2017;8.

103 Bersanelli M, Mosca E, Remondini D, et al. Methods for the integration of multi-omics data: mathematical aspects. BMC Bioinformatics 2016;17:S15 http://bmcbioinformatics. biomedcentral.com/articles/

104 Picard M, Scott-Boyer M-P, Bodein A, et al. Integration strategies of multi-omics data for machine learning analysis. Comput Struct Biotechnol J 2021;19:3735-46.

105 Lemke C, Budka M, Gabrys B. Metalearning: a survey of trends and technologies. Artif Intell Rev. 2015.

106 Sima C, Braga-Neto UM, Dougherty ER. High-Dimensional bolstered error estimation. Bioinformatics 2011;27:3056-64.

107 Ali M, Aittokallio T. Machine learning and feature selection for drug response prediction in precision oncology applications. Biophys Rev 2019;11:31-9.

108 Barla A, Jurman G, Riccadonna S, et al. Machine learning methods for predictive proteomics. Brief Bioinform 2008;9:119-28.
109 Grollemund V, Pradat Pierre-FranÃßois, Querin G, et al. Machine learning in amyotrophic lateral sclerosis: achievements, pitfalls, and future directions. Front Neurosci 2019;13. doi:10.3389/ fnins.2019.00135. [Epub ahead of print: Available from].

110 DSW H, Schierding W, Wake M, et al. Machine learning SNP based prediction for precision medicine. Front Genet [Internet] 2019;10. [Epub ahead of print: Available from].

111 Krittanawong C, Bomback AS, Baber U, et al. Future direction for using artificial intelligence to predict and manage hypertension. Curr Hypertens Rep 2018;20:75.

112 Long NP, Yoon SJ, Anh NH, et al. A systematic review on metabolomics-based diagnostic biomarker discovery and validation in pancreatic cancer. Metabolomics 2018;14:109.

113 Martinez BI, Stabenfeldt SE. Current trends in biomarker discovery and analysis tools for traumatic brain injury. J Biol Eng 2019;13. doi:10.1186/s13036-019-0145-8. [Epub ahead of print: Available from].

114 Patil S, Habib Awan K, Arakeri G, et al. Machine learning and its potential applications to the genomic study of head and neck cancerâ€”A systematic review. J Oral Pathol Med 2019;48:773-9.

115 Saini G, Mittal K, Rida P, et al. Panoptic view of prognostic models for personalized breast cancer management. Cancers 2019;11:1325.

116 Europen Council. Council conclusions on personalised medicine for patients. Off J Eur Union [Internet]. 2015;431:1-4 https://eur-lex. europa.eu/legal-content/EN/TXT/PDF/?uri=CELEX:52015XG1217( 01)\&from $=\mathrm{EN}$ 